\title{
Optimal maintenance policy of multiple parts with operating cost dependent on repair level
}

\author{
Koichi NAKADE* and Hiroaki MIKURI* \\ *Department of Architecture, Civil Engineering and Industrial Management Engineering \\ Gokiso-cho, Showa-ku, Nagoya 466-8555, Japan \\ E-mail: nakade@nitech.ac.jp
}

Received 4 September 2015

\begin{abstract}
This paper discusses properties of a preventive maintenance policy in multiple part preventive maintenance problems theoretically and numerically. A product such as a machine or an engine consists of multiple important identical parts. Parts are periodically inspected and if a part has a crack then it is either repaired or replaced with new one. If crack of a part is over the critical level then it must be replaced, because it leads to machine's breakdown and a serious problem. In some kinds of machines, using repaired parts will incur an additional operating cost, because of decrease of its performance. Repair and operation costs are also needed for maintenance. In this paper, an optimal part repair and replacement policy is developed theoretically and numerically. The model is described and formulated as a Markov decision process. For no fixed repair cost it is divided into identical single part maintenance problems, and sufficient conditions under which a threshold type policy is optimal are presented theoretically. Then the cases that optimal policies are not of threshold type are illustrated by numerical experiments.
\end{abstract}

Key words : Markov decision process, Maintenance policy, Operating cost, Repair, Replacement, Threshold type policy, Average cost

\section{Introduction}

There are many studies on maintenance policies on parts. For example, Nakagawa (1979) considers two types of repair policies, which are considering ages of parts and periodic replacement policies with minor repair. Funaki and Yoshitomi (2000) deal with a periodic preventive maintenance model with repair time minimizing the long-run expected average cost over an infinite horizon, which is the extension of minor repair models in Barlow and Hunter (1960). Surveys on preventive maintenance policies are found in Cho and Parlar (1991) and Richa et al. (2013).

Maintenance policies for multiple unit systems are important because the manufacturing system consists of multiple components in parallel or sequentially, and thus many kinds of maintenance policies have been developed. For example, Tuteja et al. (1991) deal with maintenance models for a two-unit cold-standby system with three different modes. Several kinds of models on repairmen are considered and compared. Park et al. (2009) consider a block preventive maintenance policy for a multi-unit system with periodic imperfect maintenance. Inspection and block preventive maintenance periods are decision variables and optimal maintenance policies to minimize the average cost are derived. Lai (2009) considers a two-unit parallel system with failure rate interaction. An optimal replacement policy to minimize an expected cost rate is developed. Heidergott and Farenhorst-Yuan (2010) discuss multiple component maintenance systems with a multiplegroup age-replacement policy. Various unbiased gradient estimators are derived. Nicolai and Dekker (2006) review multicomponent maintenance optimization problems, and Dekker, Nicolai and Kallenberg (2007) explain maintenance models formulated by Markov decision processes.

Most of researches on preventive and maintenance policies including above papers assume that a part or a machine 
deteriorates with its age, and it breaks down with positive probability. When some crack happens by impact of small particles or things, it leads to either repair or replacement of parts depending on the size of crack generated by impact, but the size is mostly independent of its age. In some practical problems, a relationship between ages and occurrence of crack is sometimes hard to estimate because of difficulty of the data collection, and the probability of crack occurrence depending on the age cannot be measured. In addition, in some kinds of production or machinery systems, breakdown on parts of machines incurs the serious problems. For example, if the parts of engines in cars or planes fail, the passengers in the vehicles will receive serious damage. In this system, failure of components is prohibited, and the early preventive maintenance of parts is very important, and after a predetermined period all parts will be replaced. In such a risk averse system, its safety condition implies that if a part has a crack over a safety level, it must be replaced with new one. In addition, most of papers dealing with repair assume that each part can be repaired repeatedly. As mentioned, breakdown of the important parts induces a serious problem and thus the occurrence of cracks on parts is an important factor for decision of replacement. For these parts, if a repaired part has another crack it will have to be replaced with a new part.

In literature, additional operating costs by using repaired components are not included. In some kinds of parts in processing and operating systems, the operation on machines with repaired parts needs more operating costs compared with new parts. For example, using repaired parts needs more fuels for machine working, and the necessary cost depends on the level of the repaired crack. On the other hand, if this kind of problem is considered excessively, replacement on parts will happen many times, and it will lead to high maintenance costs. To our knowledge, this kind of repair and replacement problems, such that operating costs are incurred during use of repaired items and the second damage requires replacement since the failure is prohibited, are not found in literature.

In this paper, preventive repair and replacement policies on multiple parts of one machine with operating costs are considered. After each operation period on a machine, all parts are inspected. There is a replacement level on crack, and if a part has crack of the higher level, the part must be replaced, while if it has a lower level, either repair or replacement of this part is decided. After replacement or repair of parts, the machine is operated in a deterministic period. For each replacement or repair on parts the corresponding cost is incurred. For each operation of machine, an operating cost depending on the number of repair parts and their repair levels is also incurred. After this operation, with probabilities depending on the level of repaired cracks, each part has a new crack. If repaired parts have a new crack, they must also be replaced. In this multiple part maintenance model, an optimal policy is derived for minimizing the long-run expected average cost over an infinite horizon. The model is formulated into a Markov decision process. When the operating cost is linear in the number of repaired parts and there is no fixed cost for repair, then the model is divided into identical single part maintenance problems. For the single part maintenance problem, sufficient conditions under which a threshold type policy is optimal are developed, where the threshold type policy means that all parts with new cracks of levels under a threshold are repaired and other parts are replaced with new ones. For the multiple part maintenance model which does not satisfy the conditions, optimal polices are computed by a well-known policy iteration method numerically, and it is illustrated that optimal policies may not be threshold type policies when the above conditions are not satisfied.

In the next section the part repair and replacement model is described, which is formulated into a Markov decision process in Section 3. For a linear operating cost, sufficient conditions on the optimality of threshold type policies are developed in Section 4. Numerical experiments are given in Section 5 and concluding remarks are given in Section 6.

\section{Model Description}

A machine consists of $k$ identical parts. Parts are inspected with a fixed time interval. This time interval is set as a unit period and called an operating period. At the beginning of each period, all parts are inspected whether there is a crack or not. The crack is classified with $k+1$ levels, which is called level $1,2, \ldots, k$ and $2 k+1$. A crack with level $2 k+1$ is over the safety level and a part with this level must be replaced with a new part. For each part with a crack of level $i(i=1,2, \ldots, k)$ decision is made whether it is replaced with a new part or repaired. For each part which has a repaired crack, if it has a new crack, then the part must be replaced because of the safety condition.

For each new part, after one operating period, a crack of level $i$ will occur with probability $p_{i}$ for $i=1,2, \ldots, k$ and $2 k+1$, and $p_{0}$ is set as $1-\sum_{i=1}^{k} p_{i}-p_{2 k+1}$. For a part with crack of level $i$ just repaired, it has a new crack with probability $1-q_{i}\left(i=1,2, \ldots, k, 0<q_{i}<1\right)$ during the next operating period. For a part where its crack of level $i$ has been repaired and one or more operating periods passed after its repair, which is called a part with crack of level $k+i$, 
it has a new crack with probability $1-q_{i}^{\prime}$ during the next operating period $\left(i=1,2, \ldots, k, 0<q_{i}{ }^{\prime}<1\right)$. It is assumed that the age of the part is not considered, except one period just after repair.

For each repair a repair cost $\beta$ is incurred, and for each replacement with a new part there is a replacement cost $\alpha$. When one or more repairs happen, there is a fixed repair cost $c_{x}$ which is independent of the number of repairs.

For each operating period, using the parts with repaired cracks leads to an additional operating cost. For each part with crack of level $i$ just repaired the unit operating cost is set by $c_{i}$, and for each part with crack of level $k+i$, the unit operating cost is set by $c_{i}{ }^{\prime}$ for $i=1,2, \ldots, k$. If there are $n_{i}$ parts with crack of level $i$ just repaired and $n_{i}{ }^{\prime}$ parts with repaired crack of level $k+i$ then the total one period operating cost in this period is given by

$$
C\left(n_{1}, n_{2}, \ldots, n_{k}, n_{1}^{\prime}, n_{2}^{\prime}, \ldots, n_{k}^{\prime}\right)=c_{1} n_{1}+c_{2} n_{2}+\cdots+c_{k} n_{k}+c_{1}^{\prime} n_{1}^{\prime}+c_{2}^{\prime} n_{2}^{\prime}+\cdots+c_{k}^{\prime} n_{k}^{\prime} .
$$

Here we assume the monotonicity of the operating cost, which means that the part with the bigger repaired crack needs more operating costs.

Assumption C

$$
0 \leq c_{1} \leq c_{2} \leq \cdots \leq c_{k}, 0 \leq c_{1}^{\prime} \leq c_{2}^{\prime} \leq \cdots \leq c_{k}^{\prime} .
$$

The problem is to find an optimal repair and replacement policy for minimizing the average cost.

\section{Markov Decision Process}

The model described in the previous section is formulated into a Markov decision process.

State space: At the beginning of each operating period, parts are inspected. The state consists of the numbers of parts of all crack levels. Let $m_{0}$ be the number of new parts, $m_{i}$ for $i=1,2, \ldots, k$, denote the number of parts with crack level $i$ just found at the inspection, and $m_{k+i}$ denote the number of parts with crack of level $k+i$, respectively. The notation $m_{2 k+1}$ means the number of parts which have to be replaced, which consist of parts with first crack over the safety level and parts which have been repaired before and have another new crack. Then the state space at the beginning of the period is given by

$$
S=\left\{\left(m_{0}, m_{1}, \ldots, m_{k}, m_{k+1}, \ldots, m_{2 k}, m_{2 k+1}\right) ; \sum_{i=0}^{2 k+1} m_{i}=K, m_{i} \geq 0, \quad i=0,1, \ldots, 2 k+1\right\} .
$$

For each state $s=\left(m_{0}, m_{1}, \ldots, m_{2 k}, m_{2 k+1}\right)$ in $S$, four types of decision sets $A(s)$ are considered. Note that for each part with crack of level $k+i(i=1,2, \ldots, k)$, which means that it has been repaired and there is no new crack at the current inspection, it is not replaced and used in the next operating period.

(0) Decision set 0 (Threshold policy in crack level)

In this case, the decision is made for threshold crack level $a \in\{0,1,2, \ldots, k\}$, and for parts with crack level $i$ in $\{1,2, \ldots, a\}$ they are repaired and for all parts with crack level $i$ in $\{a+1, a+2, \ldots, k\}$ they are replaced with new parts. Note that if $a=0$ then all parts are replaced and if $a=k$ then all parts are repaired. In the following, this type of policy is called a threshold type policy.

(1) Decision set 1

In this case, for each crack level $i(i=1,2, \ldots, k)$ all parts are either replaced or repaired. Thus for state $s$ its action space is

$$
A(s)=\left\{\left(a_{1}, a_{2}, \ldots, a_{k}\right) ; a_{i}=0 \text { or } 1, i=1,2, \ldots, k\right\},
$$

where $a_{i}=0$ if all parts of level $i$ are replaced, and $a_{i}=1$ if they are repaired.

(2) Decision set 2

In this case, the parts with first crack are sequenced from lower level to higher level, and first $a$ parts with lower levels are repaired and the remaining $\left(m_{1}+m_{2}+\cdots+m_{k}-a\right)$ parts are replaced with new parts. Thus

(3) Decision set 3

$$
A(s)=\left\{0,1, \ldots, \sum_{\mathrm{i}=1}^{\mathrm{k}} m_{i}\right\} .
$$

In this case, for each crack level $i, a_{i}$ parts are repaired and remaining $m_{i}-a_{i}$ parts are replaced. Thus

$$
A(s)=\left\{\left(a_{1}, a_{2}, \ldots, a_{k}\right) ; 0 \leq a_{i} \leq m_{i}, i=1,2, \ldots, k\right\} .
$$


Note that the decision set (0) is a subset of decision sets (1) and (2), which are also subsets of the decision set (3).

Intermediate State: After the decision, the intermediate state is determined. Let the number of new parts be denoted by $N_{0}$, the number of parts with crack of level $i$ just repaired is denoted by $N_{i}(i=1,2, \ldots, 2 k)$. Then the set of intermediate states is defined as follows.

$$
S^{\prime}=\left\{\left(N_{0}, N_{1}, \ldots, N_{k}, N_{k+1}, \ldots, N_{2 k}\right) ; \sum_{i=0}^{2 k} N_{i}=K, N_{i} \geq 0, i=0,1, \ldots, 2 k\right\}
$$

When the decision set $(0)$ is applied and if decision $a$ in $\{0,1,2, \ldots, k\}$ is made for state $s=\left(m_{0}, m_{1}, \ldots, m_{2 k}, m_{2 k+1}\right)$, then the intermediate state $s$ ' becomes

$$
s^{\prime}=\left(m_{0}+m_{a+1}+m_{a+2}+\cdots+m_{k}+m_{2 k+1}, m_{1}, m_{2}, \ldots, m_{a}, 0, \ldots, 0, m_{k+1}, \ldots, m_{2 k}\right) .
$$

For the decision set (1), if decision $\left(a_{1}, a_{2}, \ldots, a_{k}\right)$ is taken where $a_{i}=0$ or $1, i=1,2, \ldots, k$ for state $s=$ $\left(m_{0}, m_{1}, \ldots, m_{2 k}, m_{2 k+1}\right)$, the intermediate state $s$ ' becomes

$$
s^{\prime}=\left(m_{0}+\sum_{\left\{i: a_{i}=0\right\}} m_{i}+m_{2 k+1}, m_{1} 1\left(a_{1}=1\right), \ldots, m_{k} 1\left(a_{k}=1\right), m_{k+1}, \ldots, m_{2 k}\right),
$$

where $1(\mathrm{~A})$ is an indicator function in which $1(\mathrm{~A})=1$ if event $\mathrm{A}$ is true and 0 otherwise.

For the decision set (2), if decision $a$ in $\left\{0,1, \ldots, \sum_{i=1}^{d} m_{i}\right\}$ is taken for state $s=\left(m_{0}, m_{1}, \ldots, m_{2 k}, m_{2 k+1}\right)$, the intermediate state $s$ ' becomes

$$
s^{\prime}=\left(m_{0}+\sum_{\mathrm{i}=1}^{k} m_{i}-a+m_{2 k+1}, m_{1}, \ldots, m_{d-1}, \sum_{\mathrm{i}=1}^{d} m_{i}-a, 0, \ldots, 0, m_{k+1}, \ldots, m_{2 k}\right),
$$

where $d$ satisfies $\sum_{\mathrm{i}=1}^{d-1} m_{i}<a \leq \sum_{\mathrm{i}=1}^{d} m_{i}$.

When the decision set (3) is applied and if decision $\left(a_{1}, a_{2}, \ldots, a_{k}\right)$ is made for state $s=\left(m_{0}, m_{1}, \ldots, m_{2 k}, m_{2 k+1}\right)$, then the intermediate state $s$ ' becomes

$$
s^{\prime}=\left(m_{0}+m_{1}-a_{1}+\cdots+m_{k}-a_{k}+m_{2 k+1}, a_{1}, \ldots, a_{k}, m_{k+1}, \ldots, m_{2 k}\right) .
$$

Figure 1 shows the transition of states for decision set (3) and $k=2$.

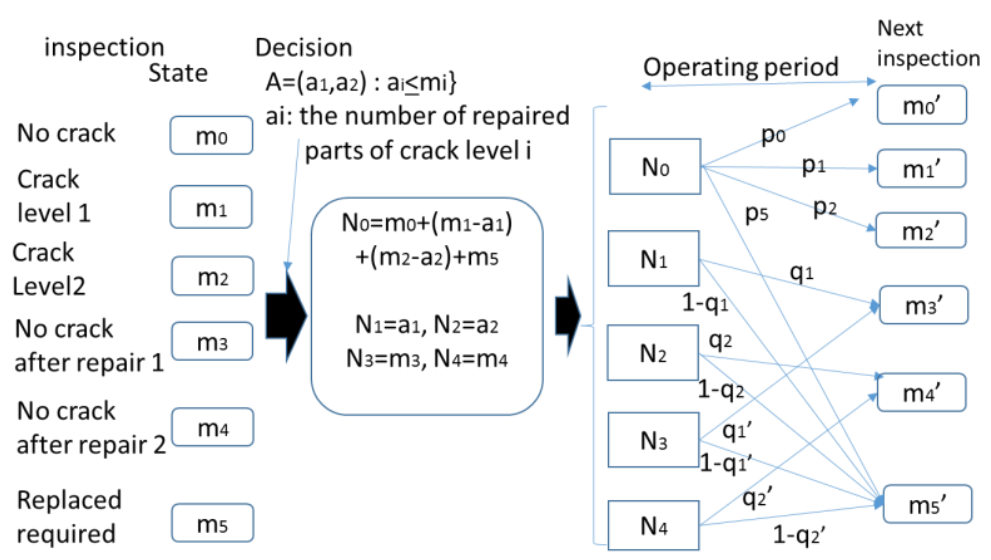

Fig. 1 State Transition (Decision set (3), $k=2$ )

Transition Probability: When an intermediate state is $\left(N_{0}, N_{1}, \ldots, N_{k}, N_{k+1}, \ldots, N_{2 k}\right)$ then the probability that the state after one operating period becomes $\left(m_{0}, m_{1}, \ldots, m_{2 k}, m_{2 k+1}\right)$, where $m_{2 k+1}=K-\sum_{\mathrm{i}=0}^{2 k} m_{i}$, is given by

$$
\begin{aligned}
& \mathrm{P}\left(\left(\left(m_{0}, m_{1}, \ldots, m_{2 k}, K-\sum_{\mathrm{i}=0}^{2 k} m_{i}\right)\right) \mid\left(N_{0}, N_{1}, \ldots, \ldots, N_{2 k}\right)\right) \\
& =f\left(\left(m_{0}, m_{1}, \ldots, m_{k}\right) \mid N_{0}\right) \times g\left(\left(m_{k+1}, \ldots, m_{2 k}\right) \mid\left(N_{1}, N_{2}, \ldots, N_{2 k}\right)\right),
\end{aligned}
$$

where the functions $f$ and $g$ are given in the following.

$$
f\left(\left(m_{0}, m_{1}, \ldots, m_{k}\right) \mid N_{0}\right)=\frac{N_{0} !}{m_{0} ! m_{1} ! \ldots m_{k} !\left(N_{0}-m_{0}-m_{1}-\cdots-m_{k}\right) !} \times p_{0}^{m_{0}} p_{1}^{m_{1}} \ldots p_{k}^{m_{k}} p_{2 k+1}^{\left(N_{0}-m_{0}-m_{1}-\cdots-m_{k}\right)},
$$




$$
\begin{aligned}
& g\left(\left(m_{k+1}, \ldots, m_{2 k}\right) \mid\left(N_{1}, N_{2}, \ldots, N_{2 k}\right)\right)=\prod_{i=1}^{k} \sum_{n=0}^{m_{k+i}} g_{i}\left(n \mid N_{i}\right) g_{k+i}\left(m_{k+i}-n \mid N_{k+i}\right), \\
& g_{i}\left(n \mid N_{i}\right)=\left\{\begin{array}{cc}
\left(\begin{array}{c}
N_{i} \\
n
\end{array}\right) q_{i}^{n}\left(1-q_{i}\right)^{N_{i}-n} & 0 \leq n \leq N_{i}, \\
0 & n>N_{i},
\end{array}\right. \\
& g_{k+i}\left(m_{k+i}-n \mid N_{k+i}\right)=\left\{\begin{array}{cl}
\left(\begin{array}{c}
N_{k+i} \\
m_{k+i}-n
\end{array}\right) q_{i}^{\prime m_{k+i}-n}\left(1-q_{i}^{\prime}\right)^{N_{k+i}-\left(m_{k+i}-n\right)} & 0 \leq m_{k+i}-n \leq N_{k+i}, \\
0 & m_{k+i}-n>N_{k+i} .
\end{array}\right.
\end{aligned}
$$

Expected Cost: The one period expected cost when action $a \in A(s)$ is taken in state $s \in S$ is denoted by $c(s, a)$. For decision set (3), the cost $c(s, a)$ is given by

where

$$
\begin{aligned}
c(s, a)= & c\left(\left(m_{0}, m_{1}, \ldots, m_{2 k}, m_{2 k+1}\right),\left(a_{1}, a_{2}, \ldots, a_{k}\right)\right) \\
= & \alpha\left(\sum_{\mathrm{i}=1}^{\mathrm{k}}\left(m_{i}-a_{i}\right)+m_{2 k+1}\right)+\beta\left(\sum_{i=1}^{k} a_{i}\right)+c_{1} a_{1}+\cdots+c_{k} a_{k}+c_{1}^{\prime} m_{k+1}+\cdots+c_{k}^{\prime} m_{2 k} \\
& +\delta\left(\left(a_{1}, a_{2}, \ldots, a_{k}\right)\right) c_{x} .
\end{aligned}
$$

$$
\delta\left(\left(a_{1}, a_{2}, \ldots, a_{k}\right)\right)=\left\{\begin{array}{rr}
0 & \left(a_{1}, a_{2}, \ldots, a_{k}\right)=(0,0, \ldots, 0) \\
1 & \text { otherwise } .
\end{array}\right.
$$

For decision sets (0), (1) and (2) the expected cost in a unit period can be defined in the similar way.

\section{Optimality of Threshold Type Policy}

In this section we discuss the optimality of threshold type policy. It is assumed that $c x=0$. That is, there is no fixed repair cost. In this case, from the model assumption, the action for each part has no effect on the transition probability and cost for other parts, and there is no additional cost depending on the decision for each part. Therefore, the stochastic process of the multidimensional states on all parts can be divided into $K$ independent processes, each of which corresponds to one part, and the action on each part is determined independently for minimizing the average cost. In the remaining of this section an optimal maintenance problem on one part is developed. For this repair and replacement problem on one part, the model is formulated into the following Markov decision process:

State Space $\mathrm{S}=\{0,1,2, \ldots, 2 k, 2 \mathrm{k}+1\}$,

Action Space $\mathrm{A}(i)=\{2\}$ for $i=0, k+1, k+2, \ldots, 2 k, \mathrm{~A}(i)=\{0,1\}$ for $i=1,2, \ldots, k$, and $\mathrm{A}(i)=\{0\}$ for $i=2 k+1$, $v(i)$ : the relative cost of state $i$ in $\mathrm{S}$,

$c(s, a)$ : one period expected cost when state is $i$ and action $a$ is taken.

Actions 0,1 and 2 mean replacement, repair and no action, respectively. Then we have the following optimality equations.

$$
\begin{gathered}
g^{*}+v(0)=\sum_{x=0}^{k} p_{x} v(x)+p_{2 k+1} v(2 k+1), \\
g^{*}+v(i)=\min \left[\begin{array}{c}
\alpha+\sum_{x=0}^{k} p_{x} v(x)+p_{2 k+1} v(2 k+1) \\
\beta+c_{i}+\left(1-q_{i}\right) v(2 k+1)+q_{i} v(k+i)
\end{array}\right] \quad i=1,2, \ldots, k \\
g^{*}+v(k+i)=c_{i}^{\prime}+\left(1-q_{i}^{\prime}\right) v(2 k+1)+q_{i}^{\prime} v(k+i) \\
g^{*}+v(2 k+1)=\alpha+\sum_{x=0}^{k} p_{x} v(x)+p_{2 k+1} v(2 k+1),
\end{gathered}
$$

where $g^{*}$ is the optimal average cost of this single part problem.

Let

$$
\begin{gathered}
D=\alpha+\sum_{x=0}^{k} p_{x} v(x)+p_{2 k+1} v(2 k+1), \\
B_{i}=\beta+c_{i}+\left(1-q_{i}\right) v(2 k+1)+q_{i} v(k+i), i=1,2, \ldots, k, \\
B_{0}=-\infty, B_{k+1}=\infty .
\end{gathered}
$$

In this model, for any deterministic policy the state becomes 0 at some epoch with probability 1 . Therefore, there is an optimal deterministic policy whose action minimizes the right hand side of equation (1) (Puterman (1994)). Thus if $B_{1} \leq$ 
$B_{2} \leq \cdots \leq B_{k}$ and $B_{i} \leq D \leq B_{i+1}$ for some $i$ in $\{0,1,2, \ldots, k-1, k\}$, then a threshold type policy with threshold level $i$ is optimal.

Note that from (2) and (3) it follows that

$$
\begin{aligned}
& v(k+i)=\frac{1}{1-q_{i}^{\prime}}\left(c_{i}^{\prime}-g^{*}\right)+v(2 k+1) \\
& v(2 k+1)=\frac{1}{1-p_{2 k+1}}\left(\alpha-g^{*}+\sum_{x=0}^{k} p_{x} v(x)\right)
\end{aligned}
$$

From (4) the inequality $B_{i} \leq B_{i+1}$ is found to be equivalent to the following inequality.

$$
\frac{q_{i+1}}{1-q_{i+1}^{\prime}} c_{i+1}^{\prime}-\frac{q_{i}}{1-q_{i}^{\prime}} c_{i}^{\prime}+\left(\frac{q_{i}}{1-q_{i}^{\prime}}-\frac{q_{i+1}}{1-q_{i+1}^{\prime}}\right) g^{*}+c_{i+1}-c_{i} \geq 0 .
$$

We discuss optimal policies under some assumptions on probabilities.

(i) Assume that the following monotonicity on probabilities on crack holds:

\section{Assumption P1}

$$
1>q_{1} \geq q_{2} \geq \cdots \geq q_{k}>0,1>q_{1}^{\prime} \geq q_{2}^{\prime} \geq \cdots \geq q_{k}^{\prime}>0 .
$$

That is, the probability that the new crack occurs is increasing in crack level $k$. In this case, from (5) and Assumption C inequality $g^{*} \geq c_{k}^{\prime}$ is a sufficient condition for a threshold policy to be optimal.

Here we derive the sufficient condition that this inequality holds. Let $N$ denote a subset of $\{1,2, \ldots, k\}$ and $f(N)$ is defined as a stationary deterministic policy such that the part with new crack of level $i$ in $N$ is repaired and the part with crack of level $i \notin N$ is replaced. The average cost under policy $f(N)$ is denoted by $g(N)$. Since there exists a stationary deterministic optimal policy, it holds that $g(N) \geq g^{*}$ for all $N \subseteq\{1,2, \ldots, k\}$ and $g\left(N^{*}\right)=g^{*}$ for some $N^{*}$. Let for $i=1, \ldots, k$

$$
\begin{gathered}
\hat{c}_{i}=\alpha+\beta+c_{i}+\frac{q_{i}}{1-q_{i}^{\prime}} c_{i}^{\prime}, \\
t_{i}=\left(1-q_{i}\right) \times 1+q_{i}\left(1+\frac{1}{1-q_{i}^{\prime}}\right)=1+\frac{q_{i}}{1-q_{i}^{\prime}} .
\end{gathered}
$$

$\hat{c}_{i}$ and $t_{i}$ mean an expected total cost incurred and an expected time interval from repair to replacement of the item of crack level $i$, respectively. Then from theory of regenerative reward processes, we have

$$
g(N)=\frac{\left(1-p_{0}\right) \alpha+\sum_{i \in N} p_{i}\left(\beta+c_{i}+\frac{q_{i}}{1-q_{i}^{\prime}} c_{i}^{\prime}\right)}{1+\sum_{i \in N} p_{i} t_{i}} .
$$

Note that if $g(N) \geq c_{k}^{\prime}$ for all $N \subseteq\{1,2, \ldots, k\}$, then $g^{*} \geq c_{k}^{\prime}$. Let

$$
A_{i}=\left(\beta+c_{i}+\frac{q_{i}}{1-q_{i}^{\prime}} c_{i}^{\prime}\right) / t_{i} \quad i=1, \ldots, k,
$$

and $A_{\min }=\min _{(i=1, \ldots, k)} A_{i}$. Then we have the following results.

Lemma 1.

Assume $\mathrm{C}$ and P1. When $\left(1-p_{0}\right) \alpha \geq c_{k}^{\prime}$ and $A_{\min } \geq c_{k}^{\prime}$, it follows that $g(N) \geq c_{k}^{\prime}$ for any $N \subseteq\{1,2, \ldots, k\}$. In particular, $g(\varnothing)=\left(1-p_{0}\right) \alpha \geq c_{k}^{\prime}$.

Lemma 2.

Assume C and P1. When $A_{\min } \geq\left(1-p_{0}\right) \alpha$, it holds that $g(N) \geq g(\varnothing)(N \neq \emptyset)$. That is, the policy such that all parts with new crack are replaced is optimal.

Note that a multiple part maintenance problem can be divided into identical single part maintenance problems, in which the above lemmas are obtained when $c_{x}=0$. Therefore the following results for the multiple part maintenance problem are obtained. 
Proposition 1.

Assume $\mathrm{C}$ and $\mathrm{P} 1$, and $c_{x}=0$.

(a) If $\left(1-p_{0}\right) \alpha \geq A_{\min } \geq c_{k}^{\prime}$, a threshold type policy is optimal.

(b) If one of the following inequalities holds, then a policy where any part with new crack is replaced with new one is optimal.

$$
\begin{aligned}
& c_{k}^{\prime} \geq A_{\min } \geq\left(1-p_{0}\right) \alpha, \\
& A_{\min } \geq c_{k}^{\prime} \geq\left(1-p_{0}\right) \alpha, \text { or } \\
& A_{\min } \geq\left(1-p_{0}\right) \alpha \geq c_{k}^{\prime} .
\end{aligned}
$$

In other cases, that is when either $c_{k}^{\prime} \geq\left(1-p_{0}\right) \alpha>A_{\min }$ or $\left(1-p_{0}\right) \alpha \geq c_{k}^{\prime}>A_{\min }$ holds, it is not clear whether a threshold type policy may be optimal or not.

(ii) In the following, the sufficient condition of the optimality of threshold policy is discussed when Assumption P1 may not hold.

Suppose that the following inequalities on probabilities hold.

Assumption P2

$$
\frac{q_{i}}{1-q_{i}^{\prime}} \geq \frac{q_{i+1}}{1-q_{i+1}^{\prime}}, \quad i=1, \ldots, k
$$

This means that the expected time interval from when a part of crack level $i$ is repaired until it is broken down, $\left(1-q_{i}\right) 0+q_{i}\left(1+\sum_{n=1}^{\infty} q_{i}^{\prime}{ }_{i}\right)=\frac{q_{i}}{1-q_{i}^{\prime}}$, is greater than that for a part of crack level $i+1$ for each $i$. Note that Assumption P2 is satisfied if Assumption P1 holds, that is, Assumption P2 is a weaker condition than P1. From (5), under assumptions $\mathrm{C}$ and $\mathrm{P} 2, g^{*} \geq c_{k}^{\prime}$ is a sufficient condition for the threshold policy to be optimal.

Inequality $g(N) \geq c_{k}^{\prime}$ is equivalent to

$$
\left(1-p_{0}\right) \alpha-c_{k}^{\prime}+\sum_{i \in N} p_{i}\left(\beta+c_{i}+\frac{q_{i}}{1-q_{i}^{\prime}} c_{i}^{\prime}-c_{k}^{\prime} t_{i}\right) \geq 0 .
$$

In the same way as case (i), we have the following result for the multiple part maintenance problem.

\section{Proposition 2}

Assume $\mathrm{C}$ and $\mathrm{P} 2$, and $c_{x}=0$.

(a) If $\left(1-p_{0}\right) \alpha \geq c_{k}^{\prime}$ and $A_{\text {min }} \geq c_{k}^{\prime}$ hold, then a threshold type policy is optimal.

(b) If $c_{1}^{\prime}=c_{2}^{\prime}=\cdots=c_{k}^{\prime},\left(1-p_{0}\right) \alpha \geq c_{k}^{\prime}$ and $\beta+c_{1} \geq c_{k}^{\prime}$, then a threshold type policy is optimal.

(c) If $\beta+c_{1} \geq\left(1-p_{0}\right) \alpha$ and $c_{1}^{\prime} \geq\left(1-p_{0}\right) \alpha$, then the policy where any part with a crack is replaced with new one is optimal.

Thus, if either condition of Propositions 1 or 2 holds, then the decision set can be restricted to decision set (0) in order to derive an optimal maintenance policy.

\section{Numerical Experiments}

In this section, we give numerical examples and demonstrate the cases where the threshold type policy is not optimal.

The multiple part maintenance problem is formulated into a Markov decision process, and a policy iteration method is applied to compute an optimal policy for a given parameter set (see policy iteration method in Puterman (1994), for example). Program was coded with C and compiled by Intel Composer XE 2011. Computations are done on a PC with Intel ${ }^{\circledR}$ Core ${ }^{\mathrm{TM}} \mathrm{i} 7-3770 \mathrm{CPU} 3.40 \mathrm{GHz}$ and RAM24.0GB. Due to restriction of memory sizes, for $k=2$, the number of parts is limited to 12 for decision set (1), for which there are 6188 states, and for decision sets (2) it is limited to 9, for which there are 2002 states. For $k=3$, the number of parts is 4 , for which there are 330 states. Computation time for deriving optimal policies is at most five minutes for decision set (1) and two minutes for decision set (2) when $k=2$.

When the parameters are set as satisfying conditions in either Proposition 1 or 2 , then optimal policies become 
threshold type policies. In fact, this is confirmed by numerical examples, thus even when the decision set (3) is applied, an optimal policy becomes the one in decision set (0).

In the following, the cases that Assumptions $\mathrm{C}$ and P1 hold but conditions of Proposition 1 are not satisfied are discussed.

Here, the part which is counted for $m_{i}$ is called 'part in level $i$ ' $(i=0,1, \ldots, 2 k+1)$.

\section{Example 1.}

The parameters are assumed to be given in the following.

$$
\begin{aligned}
& p_{0}=0.406, p_{1}=0.274, p_{2}=0.190, p_{5}=0.130, \\
& q_{1}=0.970, q_{2}=0.702, q_{1}^{\prime}=0.600, q_{2}^{\prime}=0.500, \\
& c_{1}=100000, c_{2}=101000, c_{1}^{\prime}=300000, c_{2}^{\prime}=301000, \\
& \alpha=450000, \beta=100000, c_{x}=0 .
\end{aligned}
$$

The number of parts is 12 and $k=2$. In this case, $c_{k}^{\prime}>\left(1-p_{0}\right) \alpha>A_{\min }$. Here the decision set (1) is applied. In this case, when $m_{0}=7$, then the optimal policy takes replacement for parts in level 1 and repair for parts in level 2 . The probability $q_{1}$ and $q_{1}{ }^{\prime}$ are larger compared with $q_{2}$ and $q_{2}{ }^{\prime}$, and the operating cost is almost the same between levels 1 and 2 as well as between levels 3 and 4 . Therefore, if a part in level 1 is repaired, then this part will take long time to have the next crack, and thus the operating cost on this part has to be paid for a long time. Thus the replacement is better in this case. For part in level 2, $q_{2}$ is smaller and it is better to take repair decision on this part.

Example 2.

The following parameters are considered

$$
\begin{aligned}
& p_{0}=0.406, p_{1}=0.274, p_{2}=0.190, p_{5}=0.130, \\
& q_{1}=0.748, q_{2}=0.702, q_{1}^{\prime}=0.600, q_{2}^{\prime}=0.500, \\
& c_{1}=144000, c_{2}=174000, c_{1}^{\prime}=154000, c_{2}^{\prime}=184000, \\
& \alpha=500000, \beta=100000 .
\end{aligned}
$$

The number of parts is 9 . When $c x=0$, the conditions of Proposition 1 (a) are satisfied, and thus a threshold type policy is optimal. In fact, the policy which all parts with new cracks are repaired is shown to be optimal by computation.

The case that $c x=100000$ is considered and the decision set (2) is applied. Then in many states the optimal decision is replacement of all parts, but when the number of parts in level 1 is no less than 4 and there is no parts in levels 3 and 4, it is optimal that just four parts in level 1 are repaired. All parts in level 2 are replaced. For parts in level 4, the operating cost is high, and thus replacement for parts in level 2 is better. For parts in level 1, by replacing many parts at the same time, the repair cost becomes small.

\section{Concluding Remarks}

In this paper, the optimal maintenance policy of multiple parts is discussed. There are replacement and repair costs, as well as operating cost depending on repair crack levels. For no fixed repair cost, the problem is divided to identical single part maintenance problems, and the sufficient conditions under which a threshold type policy is optimal are obtained. Thus if the conditions hold, then it is enough to determine the threshold on the repair and replacement with respect to crack levels, in order derive an optimal policy. That is, under the condition it is enough for decision maker to determine the threshold from the obtained data. If the assumption does not hold, then the cases that threshold type policy is not optimal are found by numerical experiments. When Assumptions $\mathrm{C}$ and P1 are assumed, however, the threshold type policy takes an average cost which is near to the optimal average cost in our numerical examples. That is, by considering threshold policies, near-optimal policies may be obtained.

More theoretical discussion on optimal policies is needed. In addition, as the number of possible crack levels increases, the curse of dimensionality makes computation for optimal policies impossible due to limitation of the memory size. Thus computation methods for deriving near-optimal policies need to be developed. They are left for future research.

\section{Acknowledgment}

This research is supported by Grant-in-Aid for Scientific Research(C) 25350441. The authors also appreciate 
reviewers for their valuable comments which improve this paper.

\section{References}

Barlow, R. and Hunter, L. (1960). Optimum Preventive Maintenance Policies. Operations Research, Vol. 8, pp. 90-100. Cho, D. and Parlar, M. (1991). A Survey of Maintenance Models for Multiunit Systems. European Journal of Operational Research, Vol. 51, pp. 1-23.

Dekker, R., Nicolai, R.P. and Kallenberg, L. C. M. (2007). Maintenance and Markov Decision Models. In Encyclopedia of Statistics in Quality and Reliability, F. Ruggeri and F W. Faltin eds., pp. 993-1000, Chichester, Wiley.

Funaki K. and Yoshimoto, K. (2000). Periodic Preventive Maintenance Policy with Minimal Repair at Failure Incorporating Repair Time Distribution. Journal of Japan Industrial Management Association, Vol. 51, pp. 397407.

Heidergott, B. and Farenhorst-Yan, T. (2010). Gradient Estimation for Multicomponent Maintenance Systems with AgeReplacement Policy. Operations Research, Vol. 58, pp. 706-718.

Lai, M-T. (2009). A Discrete Replacement Model for a Two-Unit Parallel System Subject to Failure Rate Interaction. Quality and Quantity, Vol. 43, pp. 471-491.

Nakagawa, T. (1979). Optimum Replacement Policies for a Used Unit. Journal of the Operations Research Society of Japan, Vol. 22, pp. 338-347.

Nicolai, R. and Dekker, R. (2006). Optimal Maintenance Policy of Multi-Component System: A Review. Complex System Maintenance Handbook, Springer, London, pp. 253-286.

Park, J. H., Lee, S. C., Hong, J. W. and Lie, C. H. (2009). An Optimal Block Preventive Maintenance Policy for a MultiUnit System Considering Imperfect Maintenance. Asia-Pacific Journal of Operational Research, Vol. 26, pp. 831847.

Puterman, M. L. (1994). Markov Decision Processes. Wiley, New York.

Richa, C., Gaur. M. and Tripathi, R. (2013). A Survey of Preventive Maintenance Planning Models, Techniques and Policies for an Ageing and Deteriorating Production Systems. HCTL Open Int. J. of Technology Innovations and Research. Vol. 3, pp. 89-107.

Tuteja, R. K., Arora, R. T. and Taneja, G. (1991). Analysis of a Two-Unit System with Partial Failures and Three Types of Repairs. Reliability Engineering and System Safety, Vol. 33, pp. 199-214. 